
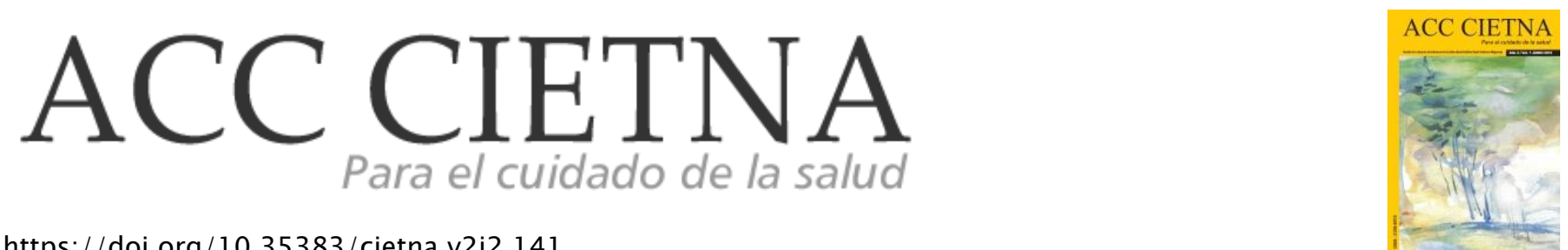

https://doi.org/10.35383/cietna.v2i2.141

\title{
El ex-sistir de ser docente de Enfermería en una universidad pública
}

\section{Morgan Zavaleta Ysabel Maltilde ${ }^{1}$, Tello Pompa Carlos Alberto ${ }^{2}$}

\section{INFORMACIÓN DEL ARTÍCULO}

Historia del artículo:

Recibido el 23 de mayo de 2015

Aceptado el 15 de octubre de 2015

\section{Palabras claves:}

Fenomenología

Ser docente de enfermería

Universidad pública

\section{RESUMEN}

Investigación cualitativa de trayectoria fenomenológica tuvo como objeto de estudio develar el significado de ser docente de enfermería, y como objetivo analizar comprensivamente el ex-sistir del docente de enfermería en una universidad pública. Se entrevistaron a diez docentes de la escuela de enfermería de la Universidad Nacional del Santa, teniendo en cuenta los criterios de selección y las consideraciones éticas y de rigor. Los hallazgos permitieron comprender el ex - sistir de ser docente de enfermería, en experiencia de su existir como profesional y persona. Se concluyó que la acción e interacción que se da entre la docente, las profesionales de enfermería, las otras docentes y estudiantes, como resultado del proceso enseñanza-aprendizaje, sumerge a la docente en un cotidiano de temor, preocupación, angustia, compromiso, responsabilidad, preparación, paciencia, también de gratificación, satisfacción, alegría, reconocimiento, con sensibilidad humana, en su papel y función docente, como profesional capaz, competente, ayudando a otro ser humano que necesita de su cuidado científico y humanístico; develando el significado de ser docente de enfermería. El temor es para el docente una situación compleja que tiene múltiples facetas, tanto por el ambiente, donde tiene que enfrentarse a la necesidad de ser apoyado y por otro lado estar preparado, lo que hace que la docente trabaje bajo tensión, sintiendo angustia si el estudiante no está bien formado.

\footnotetext{
${ }^{1}$ Licenciado en Enfermería. Enfermera, Departamento Académico de Enfermería, Universidad Nacional Del Santa, Chimbote, Perú. Email: yyy@usat.edu.pe

2 Licenciado en Enfermería. Enfermero, Escuela de Post grado. Universidad Nacional de Trujillo, Trujillo, Perú. Email: ct@hotmail.com
} 
The ex-sistir be teaching of nursing in a public university

\section{ABSTRACT}

Keywords:

Phenomenology

Being a nursing profesor

Public university
Phenomenological qualitative research that had as the object of study to reveal the meaning of being a nursing professor, and as objective to analyze comprehensively the ex-sist nursing professor at a public university. Ten professors of the school of nursing at the National University of Santa were interviewed, taking into account the selection criteria and ethical and rigor considerations.

The findings allowed us to understand the ex - sist of being a nursing professor, in her experience there as a professional and person. It was concluded that the action and interaction that occurs between professors, nurses, the other professors and students, as a result of the teachinglearning process, immerses her in everyday fear, concern,anxiety, commitment, responsibility , preparation, patience, but also of gratification, satisfaction, joy, recognition, with human sensitivity, in her role and educational function, as capable professional, competent, helping another human being who needs her scientific and humanistic care; revealing the meaning of being a nursing professor. The fear is a complex situation for the profesor that has many facets, both for the environment, where she has to face the need to be supported and on the other hand to be prepared, which makes the professor works under stress, feeling distress if the student is not well formed.

\section{Introducción}

El docente de enfermería juega un papel valioso en la formación de las futuras generaciones, orienta al estudiante a conocerse a sí mismo ontológicamente, como persona cuidadora $y$ profesional para ejercer el cuidado en la vida de otros. Tiene mucho que aprender y desarrollar en varias áreas del conocimiento; ejercita la humanidad, la tolerancia, la paciencia, la solidaridad, la reciprocidad, la confianza, la responsabilidad, y así transforma su práctica con conciencia, siempre preocupado porque el estudiante entienda la verdadera importancia de la persona, la familia, los grupos y de la vida misma. Este esfuerzo de vivencias y experiencias tienen mucha relación con la esencia humana, la cual se identifica como el ex-sistir.
El ex-sistir es para el ser humano, su esencia en esa relación que Heidegger ${ }^{1}$ señala como tal que diferencia al ser humano con otros entes $u$ otros seres en cada uno de los escenarios o ambiente donde se inserte. En ese sentido, se considera el ex-sistir femenino.

En cuanto al tema del ex-sistir docente de enfermería, hasta el momento no ha sido indagado por las tres universidades formadoras de enfermeras, que realizan investigaciones como parte de su formación profesional.

Para estudiar el significado de ser docente y entenderlo se requiere de un método que permita el estudio de los fenómenos subjetivos y describa a la docente en constante interacción con su ambiente en toda su complejidad, así como describir sus vivencias, como ellas sienten y 
expresan libremente sus diferentes formas de vida.

La presente investigación tuvo como objeto de estudio el significado de ser docente de Enfermería en una Universidad Pública; este estudio nos develó, cómo viven su experiencia como docentes, su compromiso, responsabilidad, temor a no estar preparada, su motivación para superarse, satisfacción, expresando que la experiencia profesional facilita enseñar a cuidar, teniendo como marco las buenas relaciones y el respeto.

Heidegger ${ }^{1}$ enfatiza el cuidado como una condición existencial, refiriéndose a la enseñanza del cuidado. El cuidar es fuente del propio ser, de modo que la esencia del hombre es ser cuidador de sí. Por ello, el sentido del cuidar inserta la manifestación del ser como un modo de cuidar propio del ex-sistir, dando apertura para que el otro se transforme, de tal manera que el hombre comprometa el propio ser en la búsqueda de su esencia.

Al cuidar de una persona se deben propiciar condiciones para que pueda manifestarse como ser, el cual incluye que el cuidador (docente) le posibilite apertura de su presencia a partir del propio ser, con esa postura amplía su mirada para ver las cosas del modo como estas se muestran.

Por lo anteriormente expuesto, surge la siguiente pregunta: ¿Cuál es el ex-sistir de la docente de Enfermería en la Universidad Nacional del Santa? El objetivo que guió la investigación fue analizar comprensivamente el ex-sistir de la docente de enfermería en una universidad pública.

\section{Metodología}

Investigación cualitativa de trayectoria fenomenológica, dentro de la perspectiva de Martin Heidegger, estudio que interroga al fenómeno directamente tratando de describir o llegar a su esencia existencial del ex-sistir de ser docente de enfermería en una universidad pública. El escenario fue la Escuela de Enfermería de la
Universidad Nacional del Santa - Chimbote. Los participantes fueron 10 docentes de dicha escuela, que cumplieron los siguientes criterios de selección: a) Ser docente de enfermería en condición de nombrado o contratado, b) Tener un mínimo de dos años de servicio, c) Que acepten participar voluntariamente. El tamaño de la muestra se determinó por los criterios de saturación y redundancia de los discursos.

Los datos se recolectaron mediante la entrevista. Para el proceso de recolección de la información se procedió a la interacción con las docentes de la Escuela de Enfermería, dando a conocer el objetivo de la investigación y solicitar el consentimiento informado. Después de solicitar el permiso y permiso para grabar el discurso, se le planteó la siguiente pregunta orientadora: Reláteme / cuénteme, ¿qué es para usted ser docente de enfermería en una universidad pública?

Una vez obtenido el discurso, se transcribió en texto, después, se leyó varias veces hasta encontrar su sentido. Luego se procedió a realizar la siguiente entrevista y así sucesivamente hasta obtener la saturación de datos, con diez participantes.

El procesamiento de datos, se realizó teniendo en cuenta los pasos para el análisis, según unidades de significado, éste incluye: La descripción fenomenológica, la reducción fenomenológica, interpretación vaga y mediana y el análisis hermenéutico; el cual constituye un proceso en el cual se yuxtaponen las unidades de significado encontradas en los discursos de las participantes, de tal manera que posibilitó confrontar con las ideas y conceptos de Martin Heidegger en su obra "Ser y Tiempo", lo que nos aproximó a una ontología fundamental que en este caso fue la develación del Dasein; ello implicó la determinación del ser que está oculto en el ente.

El rigor metodológico en la investigación estuvo dado por los criterios de rigor científico: 
Credibilidad, auditabilidad y aplicabilidad según Castillo $^{2}$ La Credibilidad se cumplió porque los hallazgos fueron reconocidos como reales 0 verdaderos por las personas que participaron en el estudio. Auditabilidad o confirmabilidad se efectuó porque la investigadora describió textualmente las narraciones de los informantes, esperando que otro investigador pueda seguir la "pista" al investigador original y llegue a hallazgos similares. La Aplicabilidad o transferibilidad se evidenció porque existe la posibilidad de transferir los resultados a otros contextos o grupos.

El rigor ético se cumplió por los siguientes principios: de beneficencia, a las docentes investigadas se les brindó protección contra daño psicológico y físico que pudo haber acontecido durante la investigación, brindándoles una información clara y precisa de la investigación y un ambiente tranquilo durante la entrevista. Principio de justicia, la información obtenida por las participantes se mantuvo en estricta confidencialidad. Se les hizo valer su derecho a un trato justo, y a la privacidad. Principio de respeto o dignidad humana, las docentes en forma voluntaria decidieron participaren la investigación ${ }^{3}$.

\section{Resultados, análisis y discusión}

Buscando comprender los significados que expresaron las docentes en los discursos, se procedió a su respectivo análisis con el fin de develar el sentido de ser docente de enfermería en una universidad pública. La perspectiva de Heidegger fue el referencial para interpretar comprensivamente los modos de ser que rebasaron en la experiencia concreta de lo vivido.

Se elaboraron cinco unidades de significado, las cuales reflejan la experiencia vivida por las docentes de enfermería en una universidad pública: Unidad de Significado I: El temor, dificultad y preocupación son percibidas por las enfermeras en su función docente.
Unidad de Significado II: Ser docente de enfermería es compromiso, dedicación, reto, paciencia, responsabilidad, exigencia, superación y gratificación.

Unidad de Significado III: La capacitación, el mejoramiento, la preparación de la enseñanza y las motivaciones personales son responsabilidades inherentes a la docencia.

Unidad de Significado IV: La experiencia profesional y el conocimiento compartido con el estudiante son condiciones para la enseñanza del cuidar/cuidado.

Unidad de Significado V: El Ambiente de trabajo es percibido como positivo, no obstante hay momentos difíciles y críticos.Colocar aquí los resultados análisis y discusión

Unidad de significado I: El temor, dificultad y preocupación son percibidas por las enfermeras en su función docente.

El cuidar del Sujeto del Ser-ahí estudiante, cualquiera que sean sus circunstancias es la esencia del docente.

Preocupación y ocupación que absorbe a la docente de enfermería en ese día a día de siendo, a partir de la vida cotidiana. La docente en esa cotidianidad de siendo, en sus vivencias percibe que ser docente de enfermería encierra mucha responsabilidad, compromiso para preparase, y estar junto al estudiante. El Ser-ahí en su función docente, en el proceso de cuidar al Ser - ahí que está formando, asume con coraje desafíos, ella se basa en sus conocimientos, experiencias pasadas, tiene confianza, sin embargo expresa temor.

El temor es el sentimiento de inquietud y miedo que provoca la necesidad de huir ante alguna persona o cosa, como lo manifiestan las participantes:

“...Al comienzo tuve un poco de temor, era la primera vez que ingresaba a la docencia, me 
preocupaba cómo me iba a desenvolver...". Orquídea.

"...Mi temor es que los estudiantes salgan mal, trato de mejorar...". Chavelita.

Unidad de significado II: Ser docente de enfermería es compromiso, dedicación, reto, paciencia, responsabilidad, exigencia, superación y gratificación.

Cuidar es un proceso en el que docente asume un compromiso de continuidad con el estudiante, ambos son participantes en un proceso de aprendizaje mutuo. La docente de enfermería, se enfrenta a un mundo que no es su mundo habitual, en un día a día que tiene que enfrentar junto a sus estudiantes, nuevas vivencias, experiencias, que exigen estar preparados, la preocupación que vive el ser docente yace en la preparación e intervención del estudiante en su cuidado a los otros entes humanos. Formarse pedagógicamente no solo es necesario, forma parte de la ética profesional, es compromiso que se asume con los estudiantes, y con nosotros mismos; el docente responsable conoce métodos y estrategias para lograr el aprendizaje de los(as) estudiantes (Zabalza4, tal como lo afirma las participantes:

“...Ser docente de enfermería es exigencia, es un compromiso...". Rosa.

“...Es de mucha responsabilidad, no sólo es dar al estudiante conocimientos, es darles una atención integral; verlos como personas...". Orquídea.

Unidad de significado III: La capacitación, el mejoramiento, la preparación de la enseñanza y las motivaciones personales son responsabilidades inherentes a la docencia.

El Ser - ahí, cuyo modo presencial es el ser docente de enfermería en una universidad pública, se apropia del cuidar/cuidado, ocupación, preocupación, en su mundo como docente. Siendo en el mundo, la presencia, siempre existe en cuanto siendo-con, se expresa por el cuidar y ocuparse de las/los estudiantes y preocuparse en mejorar la enseñanza, por los estudiantes en el día a día. Así lo expresan las participantes:

“...El docente debe prepararse más y más para enseñar al estudiante a dar buen cuidado, el docente debe estar actualizado para compartir con los estudiantes. Es enseñar a cuidar al ser humano y no hay que equivocarse...". Dalia.

“...Me esfuerzo por producir materiales para mejorar la enseñanza - aprendizaje...". Chavelita.

Unidad de significado IV: La experiencia profesional y el conocimiento compartido con el estudiante son condiciones para la enseñanza del cuidar/cuidado.

Ex-sistir en el mundo significa dárselas en posibilidades, ser en el mundo son las varias maneras que el existir humano o "Dasein" esta posibilitado a vivir, este "Ser en" significa familiaridad. Se refiere a la relación de la docente con las/los estudiantes, compañeros de trabajo, "ser con" es un conjuntivo fundamental del serahí, significa estar junto a algo o alguien en la presencia del otro. Lo que caracteriza el día a día de la docente, donde muestra una particularidad de modo de ser de la presencia, cuya existencia la vive en función de lo que se podría llamar un ser ahí-con, en este caso, sus estudiantes, sus compañeros de trabajo, compartiendo sus experiencias, conocimientos, brindando su ayuda, así lo manifiestan las participantes:

"La experiencia profesional me ha facilitado enseñar, la docencia te permite transmitir tus experiencias, ser comprensiva con el temor $e$ inseguridad del estudiante“. Azucena.

"...He ganado mucha experiencia he aprendido la forma de cuidar, cómo lograr una relación terapéutica de ayuda y ahora puedo transmitirlo a través de la docencia, siento que tengo mucho que dar...". Flor.

Unidad de Significado V: El ambiente de trabajo es percibido como positivo, no obstante hay momentos difíciles y críticos. 
El ser - ahí que caracteriza a la docente posibilita un "Dasein" en el modo de autenticidad o inautenticidad, vacila su disposición de todos los días, para después volverse autentica, cuando ese día se rompe con la presencia de otros, quienes requieren su apoyo, y de los que requieren de sus conocimientos, experiencia, paciencia, originando así el modo de autenticidad, donde la docente se siente apoyada, como lo expresan las participantes:

“...En el campo hospitalario y en la escuela, las relaciones son bastantes positivas, siempre trato de llevarme bien con los colegas y con todos...". Dalia.

“...Siento que las colegas me apoyan, tengo buenas relaciones con ellas...". Violeta.

La inautenticidad y la autenticidad se desdobla por el modo por el cual las docentes se relacionan entre si, quiere decir en la solicitud, que es relacionarse con docentes, estudiantes, de una manera envolvente y significativa. La solicitud tiene un carácter temporal, es en razón de la temporalidad que la solicitud puede desenvolverse en cuanto consideración y paciencia originando el modo de autenticidad y sus modos deficientes, la desconsideración, la negligencia, la indiferencia, originando el modo de inautenticidad Heidegger ${ }^{1}$ así lo expresan las participantes:

"...Observo que hay docentes egoístas, no comparten sus conocimientos sobre metodologías de enseñanza...". Rosa.

\section{Conclusiones}

Esta investigación permitió el conocimiento del ex - sistir de ser docente de enfermería en experiencia de su existir como profesional y persona, partiendo de analizar los testimonios de diez enfermeras como resultado de sus vivencias.

Considerando que el sentido metodológico de la investigación fenomenológica abarca interpretación y hermenéutica, nos atrevemos a considerar esta interpretación para docente de enfermería, una vez que, el área docente es constituida por profesionales, seres - ahí que tienen que relacionarse día a día con las demás docentes, profesionales de enfermería y estudiantes, en este caso es lo que se interpreta como: el ex-sistir de ser docente de enfermería en una universidad pública, que es nuestro objeto de estudio. Llegando a las siguientes consideraciones:

El ex-sistir del docente de enfermería está marcado por el temor, dificultad y preocupación; el cuidar del ser - ahí estudiante es su esencia. Le absorbe la preocupación y ocupación en la cotidianidad de siendo, percibe que ser docente de enfermería es de mucha responsabilidad, de compromiso para prepararse, y de estar junto al estudiante con temor a que no haga bien lo aprendido.

La relación docente - estudiante no debe ser de dominio, de intervención, y si de convivencia, de interacción y de común unión.

La formación de la enfermera(o) debe estar orientada y fundamentada en el principio de la persona que es cuidadora por virtud de su humanidad, este cuidado al estudiante debe darse en una relación de co - existencia y preocupación auténtica, para esclarecer el sentido de pensamientos, sentimientos, percepción y comportamientos, propiciando la comprensión del estudiante con ser humano ${ }^{5}$.

Ser docente de enfermería es compromiso, dedicación, reto, paciencia, responsabilidad, exigencia, superación y gratificación. La docente enfrenta junto a sus estudiantes nuevas vivencias, experiencias que requieren estar preparados, para brindar cuidado oportuno al ser-ahí que lo requiera.

La docente ante la solicitud del estudiante, se relaciona con ella/él de una manera significativa, 
con dedicación, responsabilidad; al cuidar de la/él estudiante, la docente posibilita a éste asumir sus propios caminos, crecer, madurar, conocerse a sí mismo y mejorar sus potencialidades, su autoestima, tener confianza en sí misma, produciendo en la docente satisfacción, sensación de haber cumplido, motivando en ella el interés en mantenerse actualizada, perfeccionándose ${ }^{6}$.

La docente asume que la capacitación, la preparación de la enseñanza y las motivaciones personales son responsabilidades inherentes a la docencia.

El ser-ahí, cuyo modo de la presencia es el ser docente de enfermería en una universidad pública, en su mundo docente ex-siste siendo-con, se expresa al cuidar de las/los estudiantes, se preocupa por mejorar la enseñanza, se siente motivada a prepararse. La capacidad para enseñar el cuidar incluye en la docente el autoconocimiento, solamente con el conocimiento de lo que se es, se puede mostrar de lo que se puede ser.

Las docentes de enfermería que laboran en una universidad pública le dan un significado especial a la docencia, les permite pensar que la vida es parte de un propósito, es decir, los docentes tienen un sentido de trascendencia que implica el reconocimiento que su vida se inserta en procesos más amplios y que los esfuerzos por lograrlo no serán en vano?.

Las docentes expresan que la experiencia profesional y el conocimiento compartido con la/él estudiante son condiciones para la enseñanza del cuidar/cuidado.

La docente vive existiendo en función de lo que se podría llamar un ser - ahí con, que podría ser sus estudiantes, compañero de trabajo, compartiendo sus experiencias, conocimientos, brindando ayuda.
La docente siente que tiene mucho que dar, que la experiencia profesional le ha facilitado enseñar, le da satisfacción compartir con el estudiante.

El cuidado-con, debe darse en una relación de coexistencia y preocupación auténtica, propiciando el aprendizaje del estudiante como ser humano.

Freire citado por Waldow 6 refiere que muchos seres humanos solo viven, no existen, pues no encuentran o no consiguen dar un significado a su vida. El ser humano vive el significado de su vida por medio del cuidado, ya que el cuidar, es un proceso interactivo de ayudar al otro ser humano a crecer y autorealizarse.

La docente al cuidar, en su verdadero sentido, se relaciona con el otro ser (estudiante, compañeros de trabajo), expresando su conocimiento y sensibilidad, demostrando habilidad técnica y espiritualidad, ayudándolo a crecer. El otro, en su experiencia, comparte su ser, su conocimiento, que ayudaran en el proceso de cuidar, así ambos contribuyen en el proceso de actualización y perfeccionamiento ${ }^{6}$.

Se puede concluir que un aspecto importante en la enseñanza del cuidar es la experiencia adquirida, alcanzada a través de la vivencia, de las situaciones que ocurren en lo cotidiano del cuidado, y que debe ser fortalecida con el conocimiento formal.

El ambiente de trabajo es percibido como positivo, no obstante hay momentos difíciles y críticos.

La docente en el día a día al relacionarse con estudiantes que requieren de sus conocimientos, experiencia, paciencia y con sus compañeros de trabajo de quienes recibe apoyo, origina así el modo de la autenticidad, donde la docente orienta , interviene, evalúa, cambia; sin embargo hay docentes que no sienten apoyo por parte de sus compañeros, originando en ellas el modo de inautenticidad, evidenciando por la indiferencia de su compañeros, y por su solicitud de que el docente que empieza debe ser apoyado. 
Enseñar el cuidar requiere de un ambiente propicio, un ambiente de cuidado, la institución debe garantizar personal docente con preparación, competente, un ambiente social favorable, donde los docentes, estudiantes expresen actitudes y comportamientos basados en una relación de respeto, solicitud, paciencia y consideración, que orienta a un óptimo grado de participación, satisfacción y nivel de eficiencia en el trabajo ${ }^{8}$.

La acción e interacción que se da entre la docente, las profesionales de enfermería, las otras docentes y estudiantes, como resultado del proceso enseñanza - aprendizaje, sumerge a la docente en un cotidiano de temor, preocupación, angustia, compromiso, responsabilidad, preparación, paciencia, pero también gratificación, de satisfacción, alegría, reconocimiento pero sobre todo con sensibilidad humana en su papel y función docente, como profesional capaz, competente, ayudando a otro Ser - ahí humano que está formando, que necesita de su cuidado científico y humanístico, para aprender a cuidar a otros Ser - ahí humanos que necesitan de ella/él. El temor es para el docente una situación compleja que tiene múltiples facetas, tanto por el ambiente, donde muchas veces tiene que enfrentarse a la necesidad de ser apoyado, y por otro lado estar preparado, porque los estudiantes necesitan de un docente competente, que resuelva sus necesidades de aprendizaje, hace que la docente trabaje bajo tensión, sintiendo angustia si el alumno no está bien formado.

El presente estudio fenomenológico nos permite conocer un nuevo enfoque de las experiencias vividas por los docentes con quienes luego de establecer empatía, relatan y cuentan cómo viven y sienten su experiencia como docentes, y los obstáculos que tienen que afrontar con sus compañeros docentes, profesionales de enfermería en el campo hospitalario, y estudiantes, lo cual como docentes nos ayuda a profundizar, reflexionar sobre el trato, cuidado que se brinda no sólo a los estudiantes, sino también a un compañero de trabajo, permitiendo ampliar el foco de interés hacia lo humano, teniendo en cuenta la subjetividad humana y el sentir profundo en cada uno de ellos.

Las docentes que inician la docencia sienten la necesidad de integrarse, por lo que se hace necesario implementar un programa de adaptación, con trato humano, para aliviar su desconocimiento y ser un Ser - ahí innovador y positivo.

Este trabajo de investigación me ha permitido comprender que el tiempo y el ser de los docentes, son diferentes al tiempo y ser de los profesionales de enfermería encargados de brindar su cuidado a los pacientes.

\section{Bibliografía}

1. Heidegger M. Ser e tempo. $9^{a}$ ed. Metrópolis: Vozes. Parte 1.2000 Colocar aquí la bibliografía

2. Castillo E, Vásquez, M. El rigor metodológico en la Investigación Cualitativa. Rev. Colombia Médica. Vol. $34 \mathrm{~N}^{\circ} 3.2003$

3. Velasquez, A. Metodología en investigación científica. Lima- Perú. Editorial San Marcos.1999.

4. Zabalza M. Ser profesor Universitario hoy. Rev. La Cuestión Universitaria $\mathrm{N}^{\mathrm{a}}$ 5, pp. 69 - 81. Universidad de Santiago de Compostela. España

5. Pinto N. El cuidado en el proceso enseñanzaaprendizaje. El arte y la ciencia del cuidado. Grupo de cuidado. Colombia.2002.

6. Waldow R. Cuidado: Uma Revisao Teorica. Rev. Gaucha de En fermagen. Porto Alegre 13(2): 2935. 1992.

7. Heidegger M. Ser e Tempo. $3^{\text {a }}$. Trad. Marcia Cavalcanti. Petrópolis. Vozes. 1998.

8. Lopez E. Clima Organizacional. Consultado el 20 de enero del 2012. Disponible en: http://www.monografias.com/trabajos 75 